\title{
Reversibility of chemotherapy-induced senescence is independent of autophagy and a potential model for tumor dormancy and cancer recurrence.
}

Tareq Saleh ${ }^{1}$, Emmanuel K. Cudjoe ${ }^{2}$, S. Lauren Kyte ${ }^{1}$, Scott C. Henderson ${ }^{3}$, Lynne W. Elmore ${ }^{4}$ and David A. Gewirtz ${ }^{1,5}$.

Department of Pharmacology and Toxicology

Virginia Commonwealth University

${ }^{1}$ Departments of Pharmacology, Toxicology and Medicine and Massey Cancer Center.

${ }^{2}$ Departments of Pharmacotherapy \& Outcomes Science

${ }^{3}$ Department of Anatomy and Neurobiology

${ }^{4}$ Department of Pathology

Virginia Commonwealth University

${ }^{5}$ To whom correspondence should be addressed at:

Massey Cancer Center

Virginia Commonwealth University

401 College St.

Richmond, VA 23298

Phone: 804-828-9523

Fax: 804-827-1134

Email: gewirtz@vcu.edu 


\begin{abstract}
Autophagy and senescence are both well-established responses to chemotherapy and radiation that often occur in parallel, contributing to growth arrest in tumor cells. However, it has not been established whether this growth arrest is reversible. This question was addressed using non-small cell lung cancer models exposed to the cancer chemotherapeutic drug, etoposide. Senescent cells that were sorted, identified by $\beta$-galactosidase staining and alterations in morphology, isolated by flow cytometric cell sorting based on $\mathrm{C}_{12}$ FDG staining, and real-time live microscopy were found to be capable of recovering proliferative capacity. Autophagy, monitored by vacuole formation, SQSTM1/p62 degradation, and LC3BII generation did not interfere with either the senescence arrest or proliferative recovery and was nonprotective in function (i.e. autophagy inhibition via both pharmacological and genetic strategies had negligible impact on the response to etoposide).

These observations argue against the premise that (chemotherapy-induced) senescence is irreversible and indicate that therapy-induced senescence may ultimately be a transient process in that at least a subpopulation of tumor cells can and will remain metabolically active and recover proliferative capacity independently of autophagic turnover. We therefore propose that dormant tumor cells may be capable of prolonged survival in a state of autophagy/senescence and that disease recurrence may reflect escape from this senescence-arrested state.
\end{abstract}




\section{Introduction}

Autophagy and senescence are both well-established responses to cellular stress ${ }^{1,2}$. Autophagy and senescence, whether induced by oncogene activation, chemotherapy or radiation often, if not always, occur in parallel, although we and others have determined that these responses are dissociable (i.e. senescence occurs and/or is sustained even when autophagy is suppressed $)^{3-5}$.

Autophagy, which is predominantly cell survival mechanism, has been proposed to contribute to therapy resistance and potentially to tumor dormancy ${ }^{1,6}$. With regard to tumor dormancy, this would require that some subset of the dormant tumor cell population could eventually escape from the dormant state. In contrast, senescence, whether a consequence of telomere shortening (replicative senescence), oncogene activation (oncogene-induced) or that which occurs in tumor cells in response to chemotherapy or radiation (therapy-induced senescence, accelerated or premature senescence) is generally considered to be irreversible ${ }^{7-10}$. However, it is clear that senescent cells actually retain reproductive potential since transformation and immortalization involve escape from replicative senescence ${ }^{11-13}$. A recent review published by our research group highlights studies of the capacity of cells to escape from the various forms of senescence ${ }^{14}$. With regard to therapy-induced senescence, reports by our group $^{15,16}$ as well as other laboratories have presented evidence in support of the premise that tumor cells in a state of senescence are not obligatorily in a terminally growth-arrested state ${ }^{17-26}$. These studies generally involved genetic manipulation of cell cycle regulators, such as p53 or p16 ${ }^{\text {INK4a }}$; however, it has remained uncertain whether spontaneous restoration of proliferative capacity can occur from the senescent state. Furthermore, conclusions relating to the reversibility 
of senescence have generally been based on studies in mass culture, where the origin of the replicating cells cannot be unequivocally attributed to the senescent population.

A primary finding of the current work is the capacity of etoposide-induced highly autophagic, senescent lung cancer cells, to resume cellular division, which we have previously termed proliferative recovery ${ }^{2}$. Furthermore, autophagy and senescence induced by etoposide are found to be dissociable and the capacity of the cells to recover post-drug treatment is not compromised when autophagy is inhibited either pharmacologically or genetically. Finally, the autophagy is clearly non-cytoprotective in function, as we have previously shown in other experimental models ${ }^{15,27,28}$.

Based on these findings, we propose the novel premise that therapy-induced senescence is reversible and independent of autophagic cell survival. Senescence, rather than autophagy, is likely to play a critical role in determining the nature of the tumor cell response in terms of transient growth arrest, the relative lack of cell death, and the ultimate re-emergence of the tumor cells from the growth-arrested state to regain proliferative function and contribute to disease recurrence. 


\section{Results}

Etoposide promotes growth arrest, autophagy and senescence in NSCLC cells followed by proliferative recovery.

H460 human NSCLC cells undergo growth arrest followed by proliferative recovery upon exposure to etoposide, a mainstay chemotherapeutic agent (Figure 1A, left panel). Exposure to $1 \mu \mathrm{M}$ etoposide, the concentration utilized in all subsequent studies, resulted in growth arrest for at least five days followed by proliferative recovery at approximately 7 days post-drug exposure. A similar response pattern to etoposide was also observed in the A549 NSCLC cell line (Figure 1A, right panel). Recovery of H460 cells is also shown in the colony forming assay in Figure 1B. As would have been anticipated based on the fact that etoposide promoted autophagy in A549 and U1810 NSCLC cell lines ${ }^{29}$, autophagy was also evident in the H460 cells exposed to etoposide. Figure 1C, left panel, shows the concentration-dependent formation of acridine orange-stained acidic vesicular organelles in the H460 cells 48 hours following removal of drug (also quantified by flow cytometry Figure 1C). The induction of autophagy was confirmed based on the increased formation of GFP-LC3 puncta in H460 cells (Figure 1D), as well as the increased lipidation of LC3 (conversion of LC3 from form I to form II) and the degradation of p62/SQTSM1 (Figure 1E), indicating that the autophagic process is progressing to completion (i.e. autophagic flux). 
Furthermore, and as early as 3 days after the initiation of drug exposure, H460 cells exhibit numerous features collectively indicative of senescence, specifically a flattened and enlarged appearance with abundant granulation, and histochemical staining for SA- $\beta$ galactosidase (SA- $\beta$-gal) activity (Figure 1F). Flow cytometric analysis quantifying the percentage of H460 cells expressing SA- $\beta$-gal activity at different concentrations of etoposide, relying on an established $\mathrm{C}_{12} \mathrm{FDG}$ fluorescent labeling procedure ${ }^{30}$, is presented in Figure 1G. In contrast to the promotion of senescence and autophagy, $1 \mu \mathrm{M}$ etoposide produced minimal apoptosis in the H460 cells as shown by TUNEL assay (Figure 1H) and quantification of Annexin V-positive cells by flow cytometry (Figure 1I). These data collectively suggest that etoposide induces a senescent growth arrest in H460 cells associated with robust autophagic vacuolation. Footnote 1.

Autophagy plays a non-cytoprotective role in response to etoposide in $H 460$ cells and does not interfere with the ability of cells to recover proliferative capacity.

Autophagy has historically been considered a survival response under conditions of nutrient deprivation or hypoxia, as well as a process that facilitates tumor growth and serves as a mechanism of resistance to therapy ${ }^{31,32}$. To determine whether autophagy might be protecting the H460 cells against etoposide toxicity and/or facilitating recovery following the senescent growth arrest, autophagy was suppressed using both pharmacological and genetic strategies, and the impact on sensitivity to etoposide was monitored. Cells were pretreated for 3 hours with the autophagy inhibitors chloroquine $(\mathrm{CQ}, 10 \mu \mathrm{M})$ or bafilomycin $\mathrm{A} 1$ (Baf, $5 \mathrm{nM}$ ) followed by 24 hours of exposure to etoposide in the presence of the CQ or Baf. The presence of CQ and Baf resulted in failure of lysosomal acidification, which is reflected by the yellow staining of vacuoles by acridine orange (Figure 2A); autophagy inhibition was confirmed by decreased 
degradation of p62/SQTSM1 in the presence of CQ or Baf (Figure 2B). Figure 2C shows that inhibition of autophagy did not alter sensitivity to etoposide in both MTT and clonogenic survival assays (except moderately with Baf at $1 \mu \mathrm{M}$ etoposide), indicating that the autophagy was not cytoprotective. This conclusion relating to the function of autophagy was supported by the temporal response studies presented in Figure 2D, where neither CQ nor Baf was able to alter the profile of growth arrest; furthermore, proliferative recovery occurred in the cells treated with etoposide both in the absence and presence of CQ or Baf.

Autophagy was also blocked genetically using shRNA-meditated knockdown of Atg5. Confirmation of gene silencing is shown by immunoblotting where p62/SQTSM1 levels are increased and LC3BI to LC3BII conversion is reduced (Figure 2E). Figure 2F shows that growth curves were similar in H460 cells where Atg5 was silenced as in the autophagycompetent cells. Furthermore, silencing of Atg5 did not sensitize the H460 NSCLC cells to etoposide (except for a small effect at the $0.25 \mu \mathrm{M}$ concentration, Figure 2G). In addition, inhibition of autophagy using both pharmacologic and genetic approaches did not significantly alter the extent of cell death induced by etoposide, as determined by Annexin/PI staining and FACS analysis (data not shown). It is therefore concluded that etoposide-induced autophagy in H460 cells is non-cytoprotective in function and its inhibition is not associated with increased cell death, consistent with the data presented in Figures 2D and F. These observations lead to the conclusion that autophagy plays a minor, if any, role in facilitating proliferative recovery in this system or interfering with the fate of the senescent cells.

In previous studies of radiation-induced autophagy and senescence in HCT-116 colon carcinoma cells, we demonstrated a direct correspondence between the extent of autophagy and senescence, where both responses were related to the extent of DNA damage ${ }^{15}$. However, in this 
work as well as studies of doxorubicin-induced autophagy and senescence in breast tumor cells, we report that the two responses are clearly dissociable ${ }^{5,15}$. Our studies further demonstrate that the senescence induced by etoposide in the H460 cells is independent of autophagy, as genetic silencing of autophagy failed to influence the promotion of senescence by etoposide (Figure 2H).

Proliferative recovery is associated with a decline in SA- $\beta$-galactosidase activity, entry into G1 and reduction in $21^{\text {Wafl }}$ expression.

A time course analysis following exposure of H460 (and A549 cells) to $1 \mu \mathrm{M}$ etoposide for 24 hours, based on the percentage of $\mathrm{C}_{12}$ FDG-positive cells (Figure 3A), coincides with the profile of growth arrest and proliferative recovery observed in Figure 1. Cell cycle analysis confirmed the transient accumulation of $\mathrm{H} 460$ cells in the G2 phase of the cell cycle followed by their re-entry into the G1 phase at approximately the same time where we consistently observe the restoration of growth capacity (Figure 3B). It is furthermore of importance to note that a fraction of the cell population was polyploid, which has been suggested to be required for the generation of daughter cells from senescent precursors ${ }^{17,18,33}$. A largely similar pattern is evident for both mRNA and protein levels of p21 ${ }^{\text {Waf1 }}$ (Figures 3C and D), as well as the expression of IL-6, a component of the senescence-associated secretory phenotype (SASP) that is strongly associated with DNA-damage-induced senescence ${ }^{34}$ (Figure 3D). Interestingly, while DNA damage is elevated at day 1 post-drug exposure and declines over time (based on phosphorylated $\gamma \mathrm{H} 2 \mathrm{AX}$ levels), DNA integrity is not fully restored even by day 7 (Figure 3E).

Evidence for proliferative recovery based on microscopy and live cell imaging. 
Proliferative recovery from senescent H460 cells was further monitored by staining with crystal violet, $\mathrm{C}_{12} \mathrm{FDG}$, and CFDA. As shown in Figure 4A, cells reverted from an enlarged, highly-vesicular (abundant cytoplasmic granules) morphology to a more rounded, smaller phenotype; the cells further demonstrated reduced $\mathrm{C}_{12} \mathrm{FDG}$-staining, consistent with reversion to a state of proliferation (Figure 4A, middle panel). Staining with the fluorigenic filiation tracer Vybrant CFDA also demonstrated enlarged flattened cells that gave rise to proliferating cells that retained staining by the fluorigenic tracer, consistent with the premise that these are daughter cells that emerged from the senescent cell population (Figure 4A, lower panel).

Since it is possible that the proliferating cells were not derived solely from the senescent population, cells were labeled with $\mathrm{C}_{12} \mathrm{FDG}$ followed by flow cytometric cell sorting and subsequent seeding of the highest $\mathrm{C}_{12}$ FDG-stained subpopulations ${ }^{15}$. As shown in Figure 4B, the highly $\mathrm{C}_{12}$ FDG-positive cells demonstrated restoration of proliferative capacity.

While the data generated in H460 mass cultures and senescence-enriched populations are consistent with TIS being reversible, we recognized that a careful lineage tracing approach is essential to distinguish between cancer cells that might have initially evaded etoposide-induced senescence from cells that had undergone TIS, but recovered proliferative potential. Consequently, the high $\mathrm{C}_{12}$ FDG-positive population was reseeded and monitored by live cell imaging using phase contrast microscopy. The temporal response after etoposide-induced senescence was found to be quite heterogeneous. Over the period of observation (Supplementary Videos 1, 2, and 3), the majority of the senescent cell population persisted in a growth-arrested state, while a few cells were observed to eventually surrender to stress and switch to apoptosis (or possibly mitotic catastrophe). However, occasional hypertrophic, flattened senescent cells that had remained in an arrested state were found to be capable of 
recovering the ability to divide (Figure 4C). Whether this is simply a stochastic outcome or reflective of unique characteristics of subsets in the tumor cell population, is a question that we will attempt to address in future work. Nevertheless, these studies, taken together with the data in Figure 4, appear to establish the reversibility of chemotherapy-induced senescence.

\section{Discussion}

Although the bulk of the literature has focused on the cytoprotective function(s) of autophagy, we and others have shown that in a number of studies interference with autophagy failed to alter drug or radiation sensitivity or to promote apoptosis ${ }^{15,27,28}$, as is also the case in the current work. In the case of radiation, the non-cytoprotective function of autophagy was dependent on the cells being mutant or null in $\mathrm{p} 53^{27}$. However, in the current work, it is clear that autophagy induced by etoposide in the H460 cells (p53 WT) is non-cytoprotective, an observation we were able to replicate with other cytotoxic agents (data not shown). These findings are consistent with a recent report by Eng et $\mathrm{al}^{35}$ demonstrating non-cytoprotective autophagy induced by more than 30 chemotherapeutic drugs in the A549 NSCLC cell line. Etoposide-induced non-cytoprotective autophagy did not appear to affect the profile of growth arrest and proliferative recovery, indicating that this form of autophagy is unlikely to contribute to drug sensitivity.

Studies of oncogene-induced senescence have been suggestive of a close relationship between autophagy and senescence ${ }^{36,37}$. However, the relationship has not been consistent in different experimental models ${ }^{38,39}$. In both this work as well as our own previous studies ${ }^{4,5,15}$, it is quite clear that autophagy and senescence are dissociable. 
On the other hand, senescence induction and resolution showed a more consistent pattern with growth arrest and recovery. Our laboratories previously reported on the promotion of senescence in response to chemotherapy and radiation, and furthermore provided evidence that therapy-induced senescence (TIS) may, in fact, be reversible ${ }^{14-16,40}$. Other research groups have also published data supporting the premise that TIS may be reversible ${ }^{17-26}$. The most significant finding generated by the current work, utilizing multiple and complementary approaches, is that recovery from senescence can and does occur even from a highly $\beta$-galactosidase-active population independently of autophagy; this finding has been reproduced in breast tumor cells exposed to doxorubicin (manuscript in preparation). Another important observation is that the fate of senescent cells following a single exposure to etoposide is largely heterogeneous; as is evident from the live time-dependent imaging studies, the bulk of senescent cells remain in a growth-arrested state, a few isolated cells fail to sustain cellular stress associated with DNA damage and surrendered to cell death, while a subpopulation of senescent cells recover proliferative capacity.

A number of factors may be responsible for allowing proliferative recovery to occur in this experimental system. One is the absence of functional $\mathrm{p} 16^{\mathrm{INK} 4 \mathrm{a}}$ in these cells ${ }^{41}$. While the activation of the p53-p21 axis accompanied by the senescence-associated secretory phenotype (SASP) may be sufficient to induce senescence-associated growth arrest in response to etoposide-induced DNA damage, a sustained senescence-based growth arrest is likely to be dependent upon $\mathrm{p} 16^{\mathrm{INK} 4 \mathrm{a}}$ function ${ }^{24,42}$. Since inactivation or loss of $\mathrm{p} 16$ is quite common in cancer cells ${ }^{43,44}$, reversibility of chemotherapy-induced senescence might not be an infrequent observation. The p16 ${ }^{\mathrm{INK} 4 \mathrm{a}}$-retinoblastoma protein $(\mathrm{RB})$ pathway has also been shown to be pivotal to the formation of Senescence-associated Heterochromatic Foci (SAHF), which are 
absent in this model (data not shown). SAHF might contribute to maintenance of the senescent phenotype $^{45}$.

Taken together with our previous findings relating to regrowth following radiationinduced senescence, these observations, based on enrichment of senescence and real-time microscopy, support the premise that senescence induced by chemotherapy or radiation can be reversed in tumor cells and this reversal is independently of autophagy. If this proves to be the case in tumor-bearing animals, we can speculate that senescence could be the basis for some forms of tumor dormancy. That is, it is possible to imagine that after being exposed to cycles of cytotoxic chemotherapy, a few senescent cancer cells can reside in a dormant state at distant sites and eventually resume proliferation, contributing to or being responsible for disease recurrence.

Footnote1: We were unable to detect Senescence-associated Heterochromatic Foci (SAHF), relying on histone $\mathrm{H} 3$ trimethylation lysine 9 (H3K9me3) as a classic senescenceassociated histone modification ${ }^{46}$ (data not shown). These findings are consistent with the apparent absence of global structural changes in chromatin in DAPI-stained nuclei. It is apparently not unusual that $\mathrm{H} 3 \mathrm{~K} 9 \mathrm{me} 3$-positive nuclear foci may not be evident in models of DNA damage-induced senescence ${ }^{44,45}$. 


\section{Methods}

\section{Reagents, drugs and antibodies.}

Etoposide, or VP-16-213, Sigma-Aldrich (E1383), lipofectamine (Invitrogen, 11668-019), Puromycin (Sigma-Aldrich, P8833), C ${ }_{12}$ FDG (Life Technologies, D2893), Hoechst 33342 (ThermoFisher Scientific, H1399), Vybrant CFDA (Thermo Fisher, V12883). Primary antibodies: SQSTM1/p62 (BD Biosciences, 610497), ATG5 (Cell Signaling Technology, 2630), LC3B (Cell Signaling Technology, 3868), TP53 (BD Biosciences, 554293), Cip1/p21 (BD Biosciences, 610234), GAPDH (Cell Signaling Technology, 2118). Conjugate antibodies: $\gamma \mathrm{H} 2 \mathrm{AX}$ antibody (BD Biosciences, 560445).

\section{Cell culture and drug exposure}

The wild-type (WT) TP53 H460 and A549 non-small cell lung cancer cell lines were generously provided by Dr. Richard Moran and Dr. Charles Chalfant, respectively, at Virginia Commonwealth University. The ATG5-knocked down $\mathrm{H} 460$ variant was generated in our 
laboratory: mission shRNA bacterial stocks for ATG5 were purchased from Sigma-Aldrich (TRCN00151963) and lentivirus generation was conducted in the HEK 293TN cells. The process involved cotransfection with lipofectamine with a packaging mixture of psPAX2 and pMD2.G constructs (Addgene, 12260, 12259). After 48 h, viruses shed into the media were collected and used to infect $\mathrm{H} 460$ cells under ultrasonic centrifugation for $2 \mathrm{~h}$. Selection was performed in Puromycin $(1-2 \mu \mathrm{g} / \mathrm{ml})$.

All cells were cultured in DMEM supplemented with $10 \%(\mathrm{v} / \mathrm{v})$ fetal bovine serum (Thermo Scientific, SH30066.03), $100 \mathrm{U} / \mathrm{ml}$ penicillin G sodium (Invitrogen, 15140-122), and $100 \mu \mathrm{g} / \mathrm{ml}$ streptomycin sulfate (Invitrogen, 15140-122). Cells were maintained at $37^{\circ} \mathrm{C}$ under a humidified, $5 \% \mathrm{CO}_{2}$ atmosphere at subconfluent densities.

At all etoposide concentration utilized, cells were exposed to the drug-containing medium for 24 $\mathrm{h}$, followed by replacement with fresh medium. Incubation with Chloroquine $(\mathrm{CQ}, 10 \mu \mathrm{M})$ or Bafilomycin A1 (Baf, $5 \mathrm{nM}$ ) were the pharmacological approaches utilized to interfere with lysosomal acidification and autophagosome/lysosome fusion, respectively. Cells were treated with the autophagy inhibitors for $3 \mathrm{~h}$ prior to the subsequent exposure to both etoposide and the autophagy inhibitor for an additional $24 \mathrm{~h}$ to ensure sufficient blockade of autophagy. Drugs were protected from light during handling.

\section{Growth inhibition and clonogenic survival}

Growth curves were generated by Trypan blue exclusion. Cells were seeded, treated (on day 0), and counted at the indicated time points following the removal of the drug from the medium or after sorting. For the clonogenic assay, cells were seeded, pre-treated with CQ $(10 \mu \mathrm{M})$ or Baf (5 $\mathrm{nM})$ for $3 \mathrm{~h}$, then treated with etoposide $(0.25,0.5,1.0$, or $5 \mu \mathrm{M})$ alone or in combination with 
CQ or Baf; drugs were removed and replaced with fresh media after $24 \mathrm{~h}$. Cells were incubated for 7 days, then fixed with methanol, stained with crystal violet, and counted (ColCount, Discovery Technology International).

\section{Flow cytometry and fluorescence microscopy}

All of the flow cytometry analyses were performed using BD FACSCanto II and BD FACSDiva software at the Virginia Commonwealth University Flow Cytometry Core Facility. For $\mathrm{C}_{12}$ FDG, acridine orange, Annexin V/Propidium Iodide, $\gamma \mathrm{H} 2 \mathrm{AX}$, and cell cycle analyses, 10,000 cells per replicate within the gated region were analyzed. Three replicates for each condition were analyzed in each independent experiment. Labeling procedures, gating, and analysis followed our previously published protocols with minor adjustment for the tested cell line ${ }^{5,15,47}$.

\section{Evaluation of senescence by $\beta$-galactosidase and $C_{12}$ FDG staining}

$\beta$-galactosidase labeling was performed as previously described by Dimri et $\mathrm{al}^{48}$ and in our previous publications ${ }^{5,15,47}$. Phase contrast images were taken using an Olympus inverted microscope (20X objective, Q-Color3 ${ }^{\mathrm{TM}}$ Olympus Camera; Olympus, Tokyo, Japan). The $\mathrm{C}_{12} \mathrm{FDG}$ staining protocol was adopted from Debacq-Chainiaux et $\mathrm{al}^{30}$. Cells were initially treated with Baf (100 $\mathrm{nM})$ for $1 \mathrm{~h}$ to accomplish lysosomal alkalization, followed by incubation with $\mathrm{C}_{12} \mathrm{FDG}$ in complete media for $2 \mathrm{~h}$ at $37^{\circ} \mathrm{C}$. Cells were harvested for flow cytometry and imaging (20X objective, Q-Color3 ${ }^{\mathrm{TM}}$ Olympus Camera, 488 filter, Olympus, Tokyo, Japan).

\section{Western blotting and immunofluorescence}

Western blots were performed as previously described ${ }^{47}$. Primary antibodies were used at a 1 : 1000 dilution except for GAPDH (1:8000 dilution). For immunofluorescence, at each time point, 
cells were fixed with methanol, permeabilized with $0.1 \%$ Triton $\mathrm{X}-100$, and blocked with $1 \%$ bovine serum albumin (BSA). Cells were exposed to a 1:100 dilution of p21 antibodies and incubated overnight at $4^{\circ} \mathrm{C}$, followed by exposure to the secondary antibody for $1 \mathrm{~h}$ at room temperature. After incubation, cells were mounted with DAPI and imaging was performed with an Olympus inverted microscope (20X objective, Q-Color3 ${ }^{\mathrm{TM}}$ Olympus Camera, 555 filter and UV light, Olympus, Tokyo, Japan).

\section{Promotion of apoptosis by Annexin V/ propidium iodide staining and TUNEL assay}

Quantification of apoptotic cells via flow cytometry was achieved per the manufacturer's instructions (AnnexinV-FITC apoptosis detection kit, BD Biosciences, 556547). For the terminal deoxynucleotidyl transferase dUTP nick end labeling (TUNEL) assay, adherent cells were harvested and centrifuged at 10,000 rpm for $5 \mathrm{~min}$ onto slides (Shandon Cytospin 4, Thermal Electron Corp) $48 \mathrm{~h}$ after etoposide removal. Slides were fixed with $4 \%$ formaldehyde for $10 \mathrm{~min}$ and then washed with PBS for 5 min at room temperature. The cells were then fixed with a 1:2 dilution of acetic acid and ethanol for $5 \mathrm{~min}$, followed by staining with a 1:1,000 dilution of DAPI at room temperature. Coverslips were sealed using clear nail polish and apoptosis was assessed by evaluating three fields per condition with an Olympus inverted microscope (20X objective, Q-Color3 ${ }^{\mathrm{TM}}$ Olympus Camera, 488 filter and UV light, Olympus, Tokyo, Japan).

\section{Cell Cycle Distribution by Propidium iodide (PI)}

Cells were harvested with $0.1 \%$ trypsin and neutralized with medium. After centrifugation, the cells were washed with PBS, then resuspended in a PI solution [50 $\mu \mathrm{g} / \mathrm{ml} \mathrm{PI}, 4 \mathrm{mM}$ sodium citrate, $0.2 \mathrm{mg} / \mathrm{ml}$ DNase-free RNase A, and 0.1\% Triton-X 100] for $1 \mathrm{~h}$ at room temperature, while being protected from light. Before analysis, $\mathrm{NaCl}$ was added to the cell suspensions to 
achieve a final concentration of $0.20 \mathrm{M}$. The cell suspensions were then analyzed by flow cytometry.

\section{Vybrant CFDA Cell Tracking}

Cells were stained with a $1 \%$ PBS solution containing Vybrant CFDA at a final concentration of $25 \mu \mathrm{M}$ for $30 \mathrm{~min}$. At the indicated time points, imaging was performed using an Olympus inverted microscope (20X objective, Q-Color3 ${ }^{\mathrm{TM}}$ Olympus Camera, 555 filter and UV light, Olympus, Tokyo, Japan).

\section{qRT-PCR}

RNA was isolated from cell cultures using an RNAqueous Micro Total RNA Isolation kit (Thermo Fisher Scientific) according to the manufacturer's recommendations. RT-PCR was carried out using the RETROscript kit (Ambion) to generate cDNA, followed by a standard $\mathrm{SYRB}^{\circledR}$ green-based PCR assay, as described previously (Elmore et al., ${ }^{49}$ and Sachs et al., ${ }^{50}$ ). The 2- $\Delta \Delta \mathrm{Ct}$ method was used to determine relative expression of the two senescence-associated genes.

\section{Live cell imaging}

Time lapse imaging of cells in culture was performed using a Zeiss Cell Observer microscope (Carl Zeiss Microscopy, Thornwood, NY), equipped with a Pecon stage incubator (set to $37^{\circ} \mathrm{C}$, 5\% $\mathrm{CO}_{2}$ ), an Axiocam MRm camera, and a Prior motorized XY stage (programmed to re-visit multiple selected sites in the culture dish). Phase contrast images were collected using a 10x / 
0.3 NA Plan-Neofluar objective lens at 5 min intervals over a $48 \mathrm{~h}$ period. Approximately 10 fields of view were time-lapse imaged in the culture dish for each experimental trial. Images were collected and processed using Zen software.

\section{Figure Legends}

Figure 1. Etoposide promotes growth arrest, autophagy and senescence in NSCLC cells followed by proliferative recovery. A. H460 and A549 cells were exposed to etoposide at concentrations of $0.25,0.5$, or $1 \mu \mathrm{M}$ for $24 \mathrm{~h}$ (day 0 ); cell number was determined over the indicated time points. $(\mathrm{n}=3)$. B. Colony formation of $\mathrm{H} 460$ cells following $1 \mu \mathrm{M}$ etoposide exposure for 24 h. $(n=2)$ C. Fluorescence microscopy showing concentration-dependent increase in acridine orange-stained vacuoles induced by $0.25,0.5$, and $1 \mu \mathrm{M}$ etoposide and quantification of acridine orange staining by FACS analysis in response to increasing concentrations of etoposide ( $\mathrm{n}=2$ ). Imaging and FACS were performed $48 \mathrm{~h}$ after drug removal. (20x objective). D. Fluorescence microscopy showing increased GFP-LC3 puncta in response to $1 \mu \mathrm{M}$ etoposide 
exposure. Imaging performed $48 \mathrm{~h}$ after drug removal. (20x objective) (n=2) E. Western blot showing the dose-dependent decrease in p62/SQSTM1 and the lipidation of LC3B in response to etoposide $(n=2)$. F. Senescence-associated $\beta$-galactosidase staining of H460 cells exposed to $0.25,0.5$, or $1 \mu \mathrm{M}$ etoposide (20x objective). G. Quantification of senescence based on $\mathrm{C}_{12} \mathrm{FDG}$ staining of H460 cells followed by FACS analysis. Staining and analysis were performed $48 \mathrm{~h}$ after drug removal (day 3) (n=3). H. TUNEL assay of H460 cells 48 h following etoposide $(1 \mu \mathrm{M})$ removal. I. Percentage of apoptotic H460 cells following \# h exposure to $1 \mu \mathrm{M}$ etoposide was determined by Annexin V immunolabeling and FACS at the same time point. Data are expressed as standard error of the mean (SEM) for " $n$ " independent experiments.

Figure 2. Autophagy plays a "non-cytoprotective" role in response to etoposide in $\mathbf{H 4 6 0}$ cells and does not interfere with the ability of cells to recover proliferative capacity. A. Fluorescence microscopy showing failure of lysosomal acidification following CQ (10 $\mu \mathrm{M})$ or Baf $(5 \mathrm{nM})$ co-treatment with $1 \mu \mathrm{M}$ etoposide. Cells were pretreated with CQ and Baf followed by an additional $24 \mathrm{~h}$ with etoposide. Images were taken $48 \mathrm{~h}$ after drug removal (20x objective). Nuclei stained with Hoechst 33342. $(n=2)$. B. Western blot showing autophagy blockade by CQ $(10 \mu \mathrm{M})$ and Baf $(5 \mathrm{nM})$ based on levels of p62/SQSTM1 (n=3). C. Left panel. MTT assay showing influence of CQ $(10 \mu \mathrm{M})$ or Baf $(5 \mathrm{nM})$ on sensitivity of H460 cells to etoposide. Cells were pretreated with $\mathrm{CQ}$ or Baf for $3 \mathrm{~h}$ followed by co-treatment with etoposide for $24 \mathrm{~h}$. Absorbance was measured $72 \mathrm{~h}$ after drug removal and replacement with fresh medium $(\mathrm{n}=3)$. Bars represent mean \pm SD absorbance values relative to untreated control. C. Right panel. Clonogenic survival assay showing influence of CQ $(10 \mu \mathrm{M})$ or Baf $(5 \mathrm{nM})$ on sensitivity of H460 cells to etoposide ( $\mathrm{n}=2$ ). Cells were pretreated with CQ or Baf for $3 \mathrm{~h}$ followed by co- 
treatment with etoposide for $24 \mathrm{~h}$. Colonies were counted 7 days following removal of drugs and replacement with fresh medium. Bars represent mean survival \pm SD relative to untreated controls $(\alpha=0.05 / 3, * p<0.016)$. D. Viable H460 cell number was determined at the indicated days following etoposide exposure in combination with CQ $(10 \mu \mathrm{M})$ or Baf $(5 \mathrm{nM}) \quad(\mathrm{n}=2)$. E. Western blot following shRNA-mediated knockdown of Atg5 (n=2). F. Temporal response to etoposide in parental H460 cells and H460 cells with knockdown of Atg5 (n=2). G. Clonogenic survival assay comparing sensitivity of shControl and shAtg5 H460 cells in response to multiple etoposide concentrations. Bars represent mean survival $\pm \mathrm{SD}$ relative to untreated controls $(\alpha=0.05 / 3, * p<0.016)(\mathrm{n}=2)$. H. Percent senescence based on $\mathrm{C}_{12}$ FDG staining at day 3 postetoposide exposure in shControl cells and cells infected with shAtg5. Unless stated otherwise, data are expressed as standard error of the mean (SEM) for " $n$ " independent experiments.

Figure 3. Proliferative recovery is associated with a decline in SA- $\beta$-galactosidase, entry into $\mathbf{G 1}$ and reduction in $\mathbf{p 2 1}{ }^{\text {Waf1 }}$ expression. A. $\mathrm{C}_{12}$ FDG staining of H460 and A549 cells following $24 \mathrm{~h}$ exposure to $1 \mu \mathrm{M}$ etoposide followed by FACS analysis over the indicated time points $(n=3)$. B. Cell cycle distribution after exposure of $H 460$ cells to $1 \mu \mathrm{M}$ etoposide $(\mathrm{n}=3)$. C. Western blot showing the induction and decline of p21 Waf1 over time in H460 cells following $1 \mu \mathrm{M}$ etoposide and immunofluorescence labeling for $\mathrm{p} 21^{\text {Waf1 }}$. DNA stained with DAPI (20x objective) (n=2). D. qRT-PCR to monitor expression of IL-6 and $p 21^{\text {Wafl }}$ genes following $1 \mu \mathrm{M}$ etoposide exposure $(n=2)$. E. Induction and repair of DNA damage in etoposide-treated H460 cells based on intensity of phosphorylated $\gamma-\mathrm{H} 2 \mathrm{AX}$ fluorescence quantified by flow cytometry. $(n=3)$. Data are expressed as standard error of the mean (SEM) for " $n$ " independent experiments. 
Figure 4. Etoposide-induced senescence in $\mathbf{H 4 6 0}$ cells can be reversible. A. H460 cells were treated with $1 \mu \mathrm{M}$ etoposide for $24 \mathrm{~h}$. Upper panel. Crystal violet on days 3, 5, and 7 . Middle panel. $\mathrm{C}_{12} \mathrm{FDG}$ staining on days 3, 5, and 7. Lower panel. Vybrant CFDA staining where cells were stained on day 3 followed by microscopy on days 4,5 , and 6 . DNA stained with Hoechst 33342. Fields are representative of separate experimental events. $(20$ x objective) $(n=2)$ B. High$\mathrm{C}_{12} \mathrm{FDG}$ positive cells were reseeded and monitored for colony formation. Colonies stained with crystal violet at day 15 after etoposide exposure (day 12 after sorting).. C. Time-lapse live-cell microscopy of H460 high C12FDG-positive cells performed for 40 h, 5 days following flow cytometry-based sorting. Arrows indicate mitotic events. Data are expressed as standard error of the mean (SEM) for " $n$ " independent experiments.

Supplementary Videos 1, 2 and 3. H460 cells were exposed to etoposide ( $1 \mu \mathrm{M}$ for 24 hours) and then sorted by flow cytometry based on $\mathrm{C}_{12} \mathrm{FDG}$ staining. Only the high $\mathrm{C} 12 \mathrm{FDG}$-positive cells were reseeded and monitored for 40 h, 5 days following flow cytometry-based sorting. Senescent cells exhibited heterogeneous fates; where many remained arrested, some restored mitotic capacity.

\section{Acknowledgments}

This work was supported by the Office of the Assistant Secretary of Defense for Health Affairs through the Breast Cancer Research Program [grant no. W81XWH-14-1-0088 (DAG)], Massey Center Support Grant P30 CA016059 and NIH grant \# RCA206028, 
The authors would like to thank Dr. Khushboo Sharma for assistance with generating the shControl, shAtg5 and GFP-LC3 H460 cells and Dr. Moureq Alotaibi for establishing the $\mathrm{C}_{12}$ FDG-based cell sorting protocol with the help of Julie Farnsworth. 


\section{Bibliography}

1. Yang ZJ, Chee CE, Huang S, Sinicrope FA. The role of autophagy in cancer: therapeutic implications. Mol Cancer Ther. 2012;10(9):1533-1541. doi:10.1158/1535-7163.MCT-110047.The.

2. Gewirtz DA. Autophagy, senescence and tumor dormancy in cancer therapy. Autophagy. 2009;5(8):1232-1234. doi:10.4161/auto.5.8.9896.

3. Gewirtz DA. Autophagy and Senescence in Cancer Therapy. J Cell Physiol. 2014;229:69. doi:10.1002/jcp.24420.

4. Gewirtz DA. Autophagy and senescence: A partnership in search of definition. Autophagy. 2013;9(5):808-812. doi:10.4161/auto.23922.

5. Goehe RW, Di X, Sharma K, et al. The Autophagy-Senescence Connection in Chemotherapy: Must Tumor Cells (Self) Eat Before They Sleep? J Pharmacol Exp Ther. 2012;343(3):763-778.

6. Lu Z, Luo RZ, Lu Y, et al. The tumor suppressor gene ARHI regulates autophagy and tumor dormancy in human ovarian cancer cells. J Clin Invest. 2008;118(12):3917-3929. doi:10.1172/JCI35512.

7. Hayflick L, Moorhead PS. The Serial Cultivation of Human Diploid Cell Strains. Exp Cell Res. 1961;25:585-621.

8. Di Leonardo A, Linke SP, Clarkin K, Wahl GM. DNA damage triggers a prolonged p53dependent G1 arrest and long-term induction of Cip1 in normal human fibroblasts. Genes Dev. 1994;8:2540-2551. 
9. Serrano M, Lin AW, McCurrach ME, Beach D, Lowe SW. Oncogenic ras Provokes Premature Cell Senescence Associated with Accumulation of p53 and p16 INK4a. Cell. 1997;88:593-602.

10. Campisi J, d'Adda di Fagagna F. Cellular senescence: when bad things happen to good cells. Nat Rev Mol Cell Biol. 2007;8:729-740. doi:10.1038/nrm2233.

11. Shay JW, Wright WE. Senescence and immortalization: role of telomeres and telomerase. Carcinogenesis. 2005;26(5):867-874. doi:10.1093/carcin/bgh296.

12. Reddel RR. The role of senescence and immortalization in carcinogenesis. Carcinogenesis. 2000;21(3):477-484. doi:10.1093/carcin/21.3.477.

13. Peeper DS, Dannenberg JH, Douma S, te Riele H, Bernards R. Escape from premature senescence is not sufficient for oncogenic transformation by Ras. Nat Cell Biol. 2001;3:198-203. doi:10.1038/35055110.

14. Chakradeo S, Elmore LW, Gewirtz DA. Is Senescence Reversible? Curr Drug Targets. 2016;17(4):460-466. doi:http://dx.doi.org/10.2174/1389450116666150825113500.

15. Alotaibi M, Sharma K, Saleh T, Povirk LF, Hendrickson EA, Gewirtz DA. Radiosensitization by PARP Inhibition in DNA Repair Proficient and Deficient Tumor Cells: Proliferative Recovery in Senescent Cells. Radiat Res. 2016;185:229-245. doi:10.1667/RR14202.1.

16. Elmore LW, Di X, Dumur C, Holt SE, Gewirtz DA. Evasion of a Single-Step, Chemotherapy-Induced Senescence in Breast Cancer Cells: Implications for Treatment Response. Cancer Ther Clin. 2005;11(7):2637-2643. 
17. Puig P-E, Guilly M-N, Bouchot A, et al. Tumor cells can escape DNA-damaging cisplatin through DNA endoreduplication and reversible polyploidy. Cell Biol Int. 2008;32:10311043. doi:10.1016/j.cellbi.2008.04.021.

18. Wang Q, Wu PC, Dong DZ, et al. Polyploidy road to therapy-induced cellular senescence and escape. Int J Cancer. 2013;132:1505-1515. doi:10.1002/ijc.27810.

19. Michishita E, Nakabayashi K, Ogino H, Suzuki T, Fujii M, Ayusawa D. DNA Topoisomerase Inhibitors Induce Reversible Senescence in Normal Human Fibroblasts. Biochem Biophys Res Commun. 1998;253:667-671.

20. Roberson RS, Kussick SJ, Vallieres E, Chen SJ, Wu DY. Escape from Therapy-Induced Accelerated Cellular Senescence in p53-Null Lung Cancer Cells and in Human Lung Cancers. Cancer Res. 2005;65(7):2795-2803.

21. Chitikova Z V, Gordeev SA, Bykova T V, Svetlana G, Pospelov VA, Pospelova T V. Sustained activation of DNA damage response in irradiated apoptosis-resistant cells induces reversible senescence associated with mTOR downregulation and expression of stem cell markers. Cell Cycle. 2014;13(9):1424-1439. doi:10.4161/cc.28402.

22. Jackson JG, Pant V, Li Q, et al. p53-Mediated Senescence Impairs the Apoptotic Response to Chemotherapy and Clinical Outcome in Breast Cancer. Cancer Cell. 2012;21:793-806. doi:10.1016/j.ccr.2012.04.027.

23. Dirac AMG, Bernards R. Reversal of Senescence in Mouse Fibroblasts through Lentiviral Suppression of p53. J Biol Chem. 2003;278(14):11731-11734.

doi:10.1074/jbc.C300023200. 
24. Beausejour CM, Krtolica A, Galimi F, et al. Reversal of human cellular senescence: roles of the p53 and p16 pathways. Eur Mol Biol Organ J. 2003;22(16):4212-4222.

25. Morton JP, Timpson P, Karim SA, et al. Mutant p53 drives metastasis and overcomes growth arrest/senescence in pancreatic cancer. Proc Natl Acad Sci U S A. 2010;107(1):246-251. doi:10.1073/pnas.0908428107.

26. Boquoi A, Arora S, Chen T, Litwin S, Koh J, Enders GH. Reversible cell cycle inhibition and premature aging features imposed by conditional expression of p16Ink4a. Aging Cell. 2015;14:139-147. doi:10.1111/acel.12279.

27. Chakradeo S, Sharma K, Alhaddad A, et al. Yet another function of p53 - the switch that determines whether radiation-induced autophagy will be cytoprotective or nonprotective: Implications for autophagy inhibition as a therapeutic strategy. Mol Pharmacol. 2015;87:803-814. doi:10.1124/mol.114.095273.

28. Saleh T, Cuttino L, Gewirtz DA. Autophagy is not uniformly cytoprotective: a personalized medicine approach for autophagy inhibition as a therapeutic strategy in nonsmall cell lung cancer. BBA - Gen Subj. 2016;1860:2130-2136.

doi:10.1016/j.bbagen.2016.06.012.

29. Kaminskyy VO, Piskunova T, Zborovskaya IB, Tchevkina EM, Zhivotovsky B. Suppression of basal autophagy reduces lung cancer cell proliferation and enhances caspase-dependent and -independent apoptosis by stimulating ROS formation. Autophagy. 2012;8(7):1032-1044. doi:10.4161/auto.20123.

30. Debacq-Chainiaux F, Erusalimsky JD, Campisi J, Toussaint O. Protocols to detect senescence-associated beta-galactosidase (SA-betagal) activity, a biomarker of senescent 
cells in culture and in vivo. Nat Protoc. 2009;4(12):1798-1806.

doi:10.1038/nprot.2009.191.

31. Janku F, McConkey DJ, Hong DS, Kurzrock R. Autophagy as a target for anticancer therapy. Nat Rev Clin Oncol. 2011;8:528-539. doi:10.1038/nrclinonc.2011.71.

32. Maiuri MC, Zalckvar E, Kimchi A, Kroemer G. Self-eating and self-killing: crosstalk between autophagy and apoptosis. Nat Rev Mol Cell Biol. 2007;8:741-752. doi:10.1038/nrm2239.

33. Mosieniak G, Sliwinska MA, Alster O, et al. Polyploidy Formation in DoxorubicinTreated Cancer Cells Can Favor Escape from Senescence. Neoplasia. 2015;17(12):882893. doi:10.1016/j.neo.2015.11.008.

34. Coppé J-P, Desprez P-Y, Krtolica A, Campisi J. The senescence-associated secretory phenotype: the dark side of tumor suppression. Annu Rev Pathol. 2010;5:99-118. doi:10.1146/annurev-pathol-121808-102144.

35. Eng CH, Wang Z, Tkach D, et al. Macroautophagy is dispensable for growth of KRAS mutant tumors and chloroquine efficacy. Proc Natl Acad Sci U S A. 2016;113(1):182-187. doi:10.1073/pnas.1515617113.

36. Narita M, Young ARJ, Narita M. Autophagy facilitates oncogene-induced senescence. Autophagy. 2009;5(7):1046-1047. doi:10.4161/auto.5.7.9444.

37. White E, Lowe SW. Eating to exit: Autophagy-enabled senescence revealed. Genes Dev. 2009;23(7):784-787. doi:10.1101/gad.1795309.

38. Cuervo AM, Dice JF. Age-related decline in chaperone-mediated autophagy. J Biol Chem. 
2000;275(40):31505-31513. doi:10.1074/jbc.M002102200.

39. Young ARJ, Narita M, Ferreira M, et al. Autophagy mediates the mitotic senescence transition. Genes Dev. 2009;23:798-803. doi:10.1101/gad.519709.

40. Gewirtz DA, Holt SE, Elmore LW. Accelerated senescence: An emerging role in tumor cell response to chemotherapy and radiation. Biochem Pharmacol. 2008;76:947-957. doi:10.1016/j.bcp.2008.06.024.

41. Kamb A, Gruis NA, Weaver-Feldhaus J, et al. A cell cycle regulator potentially involved in genesis of many tumor types. Science (80- ). 1994;264:436-440. doi:10.1126/science.8153634.

42. Herbig U, Jobling WA, Chen BPC, Chen DJ, Sedivy JM. Telomere shortening triggers senescence of human cells through a pathway involving ATM, p53, and p21CIP1, but not p16INK4a. Mol Cell. 2004;14(4):501-513. doi:10.1016/S1097-2765(04)00256-4.

43. Kratzke RA, Greatens TM, Rubins JB, et al. Rb and p16(INK4a) expression in resected non-small cell lung tumors. Cancer Res. 1996;56(15):3415-3420.

44. Narita M, Nun S, Heard E, et al. Rb-Mediated Heterochromatin Formation and Silencing of E2F Target Genes during Cellular Senescence. Cell. 2003;113:703-716.

45. Kosar M, Bartkova J, Hubackova S, Hodny Z, Lukas J, Bartek J. Senescence-associated heterochromatin foci are dispensable for cellular senescence, occur in a cell type- And insult-dependent manner, and follow expression of p16ink4a. Cell Cycle. 2011;10(3):457468. doi:10.4161/cc.10.3.14707.

46. Di Micco R, Sulli G, Dobreva M, et al. Interplay between oncogene-induced DNA 
damage response and heterochromatin in senescence and cancer. Nat Cell Biol. 2011;13(3):292-302. doi:10.1038/ncb2170.

47. Sharma K, Goehe RW, Di X, et al. A novel cytostatic form of autophagy in sensitization of non-small cell lung cancer cells to radiation by vitamin D and the vitamin D analog, EB 1089. Autophagy. 2014;10(12):2346-2361. doi:10.4161/15548627.2014.993283.

48. Dimri GP, Lee X, Basile G, et al. A biomarker that identifies senescent human cells in culture and in aging skin in vivo. Proc Natl Acad Sci U S A. 1995;92:9363-9367. doi:DOI 10.1073/pnas.92.20.9363.

49. Elmore LW, Rehder CW, Di X, et al. Adriamycin-induced senescence in breast tumor cells involves functional p53 and telomere dysfunction. J Biol Chem. 2002;277(38):35509-35515. doi:10.1074/jbc.M205477200.

50. Sachs PC, Francis MP, Zhao M, et al. Defining essential stem cell characteristics in adipose-derived stromal cells extracted from distinct anatomical sites. Cell Tissue Res. 2012;349:505-515. doi:10.1007/s00441-012-1423-7. 


\section{Reversibility of chemotherapy- induced senescence is independent of autophagy and a potential model for tumor dormancy and cancer recurrence.}

Tareq Saleh, Emmanuel K. Cudjoe, S. Lauren Kyte, Scott Henderson, Lynne W. Elmore and David A. Gewirtz. 


\section{Figure 1}

A.
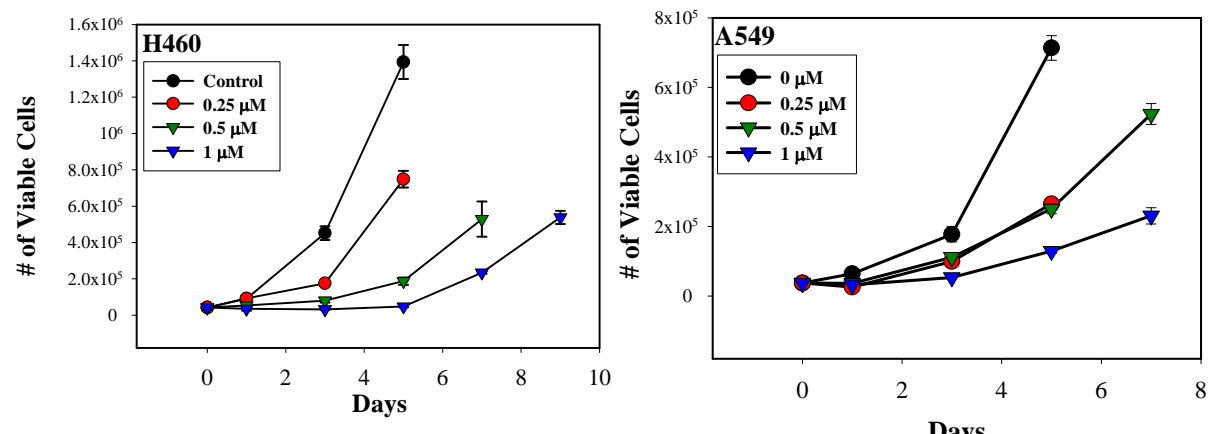

B.

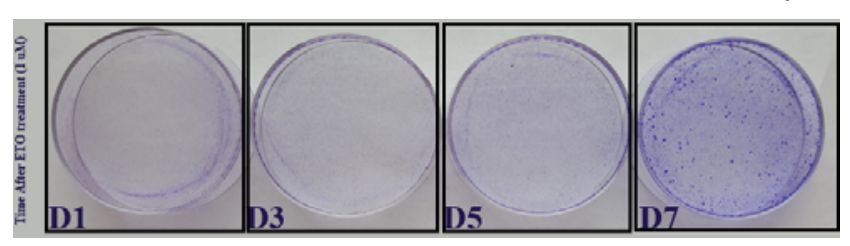

C.
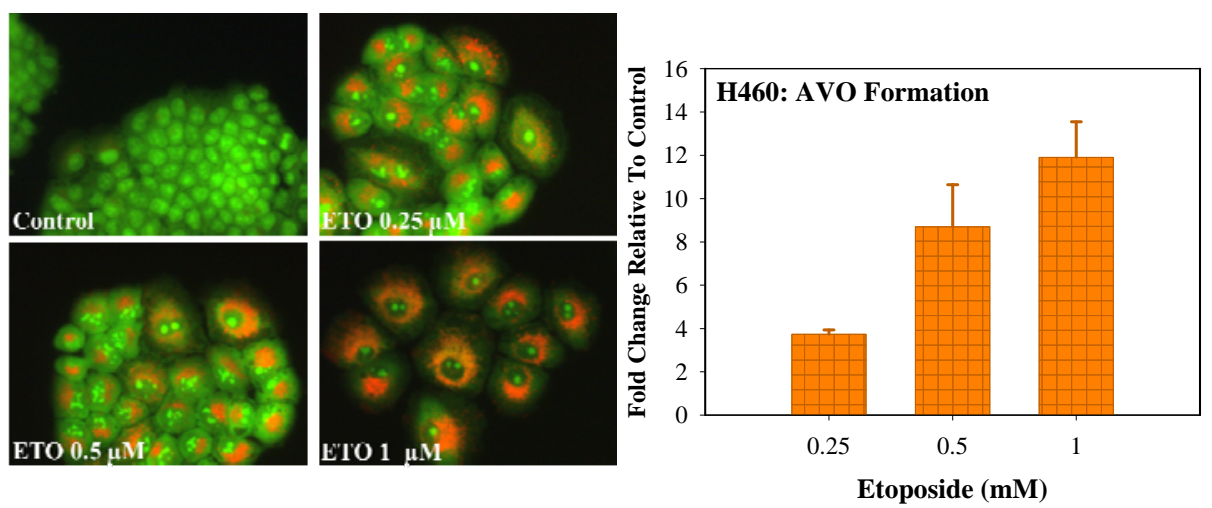

D.

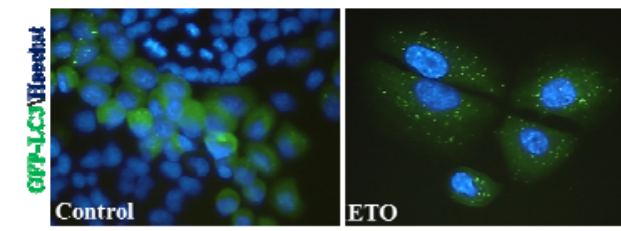

F.

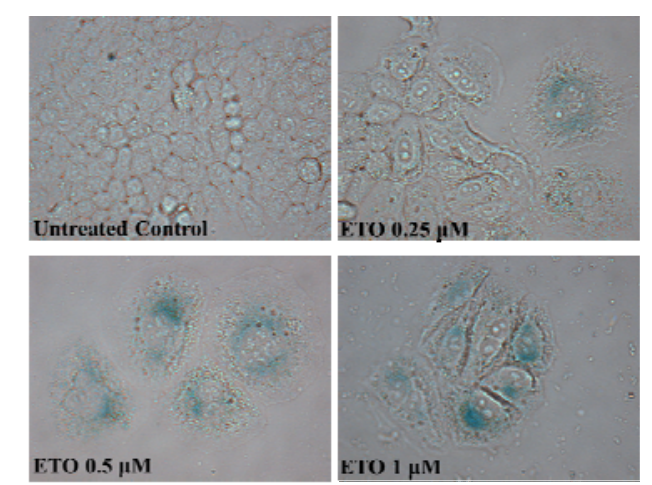

H.

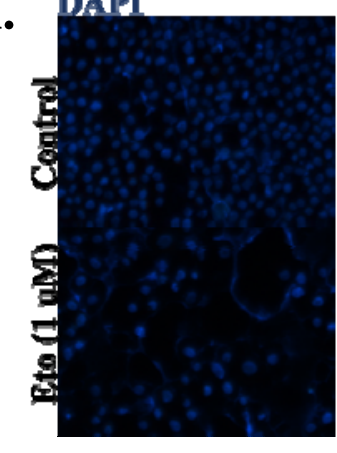

E.

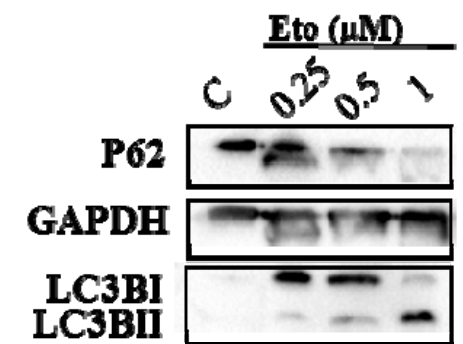

G.
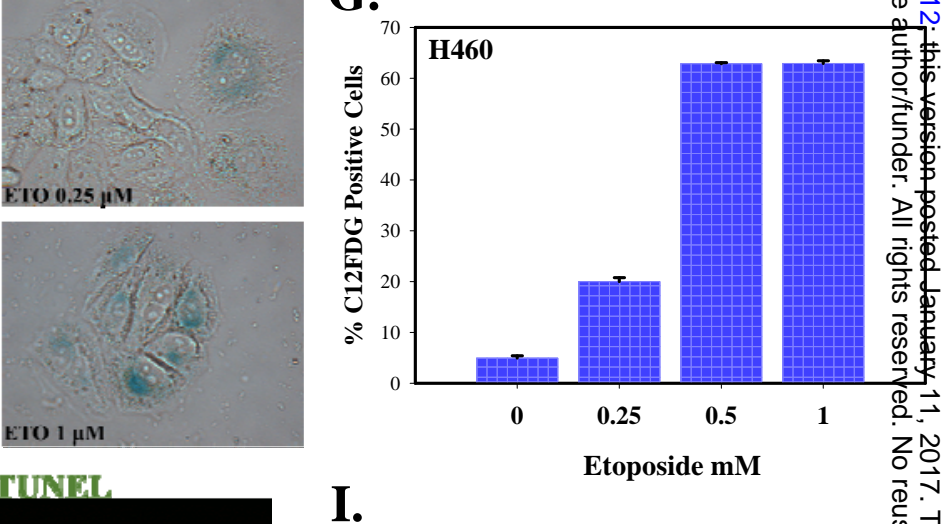

I.

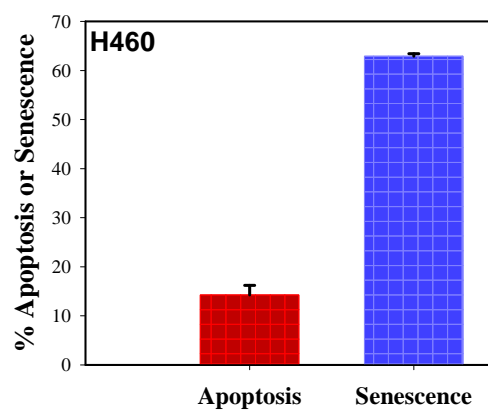




\section{FIGURE 2}

A.

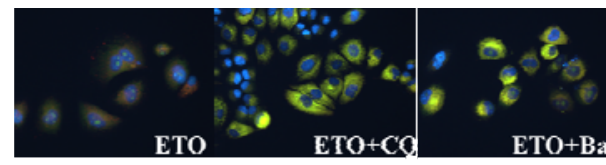

C.

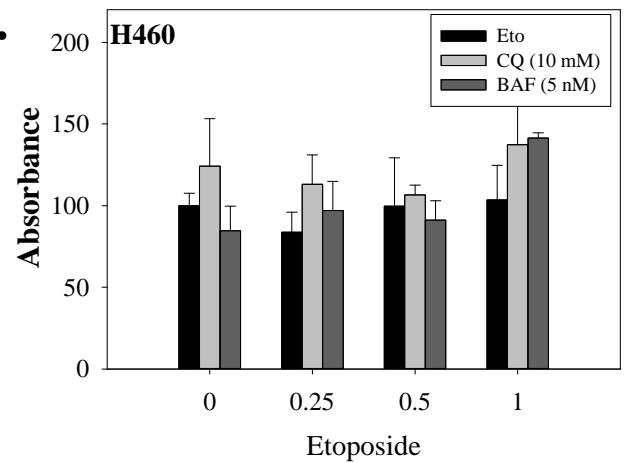

D.

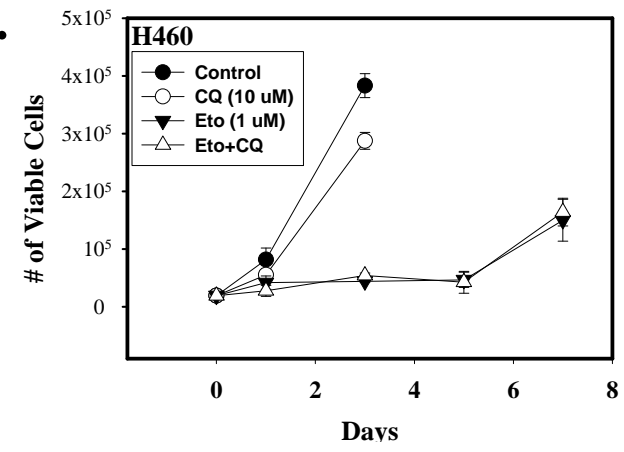

B. 12

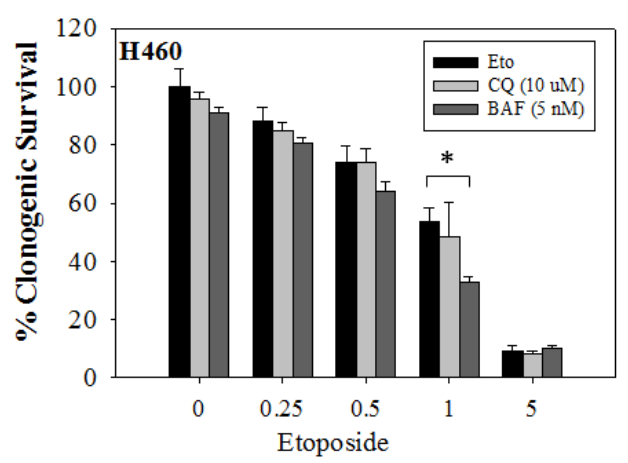

G.

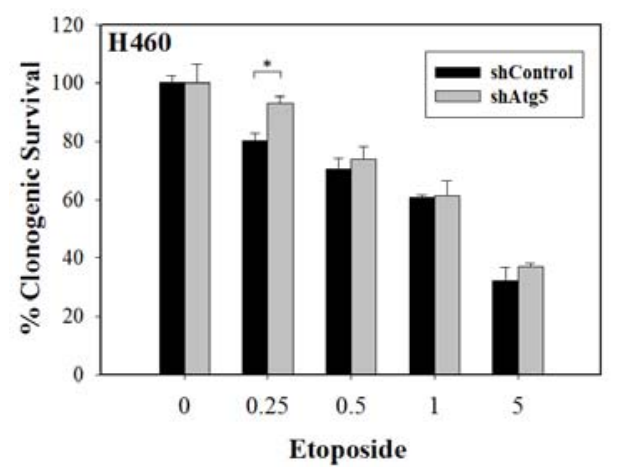

A.

p6

GAPDH

LC3B I

LC3B II
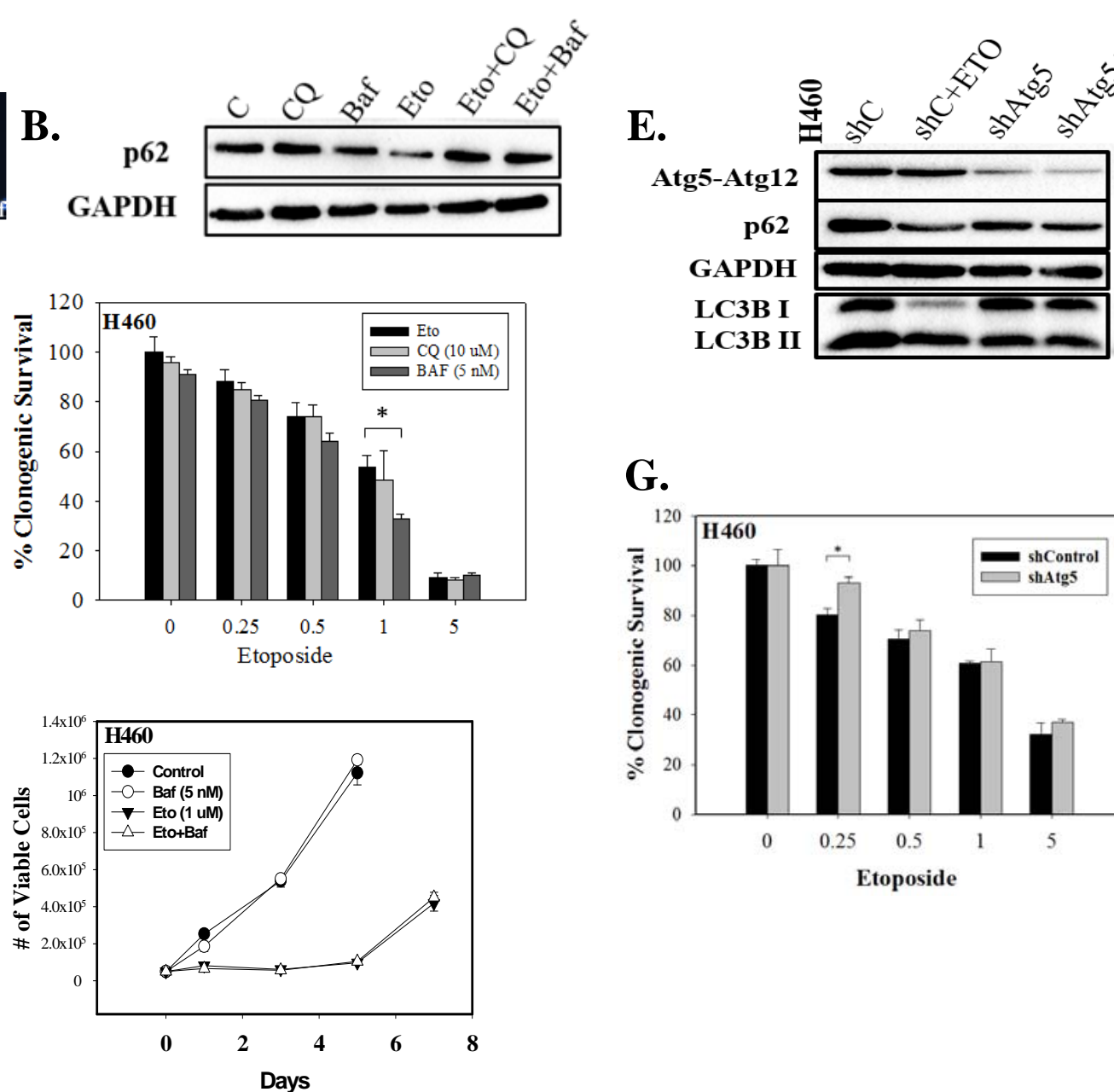

F.

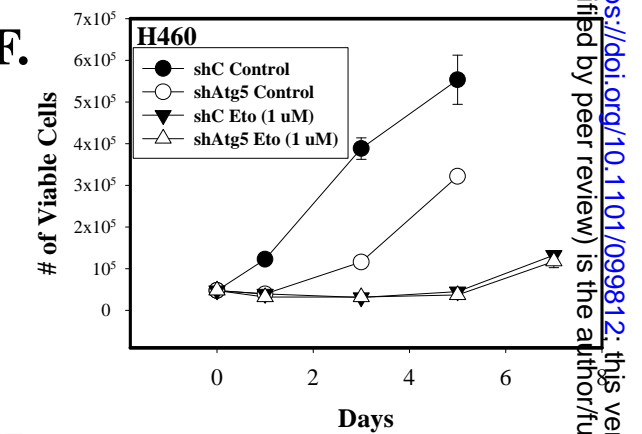

H.

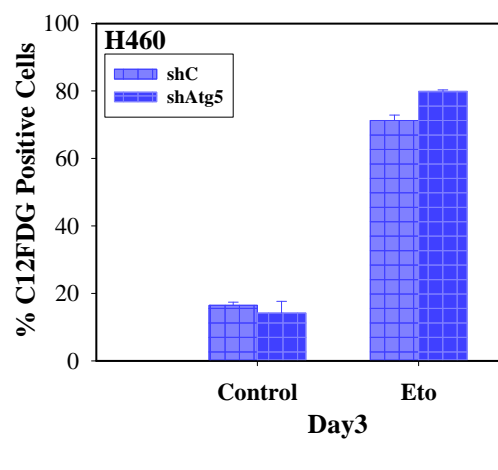




\section{FIGURE 4}

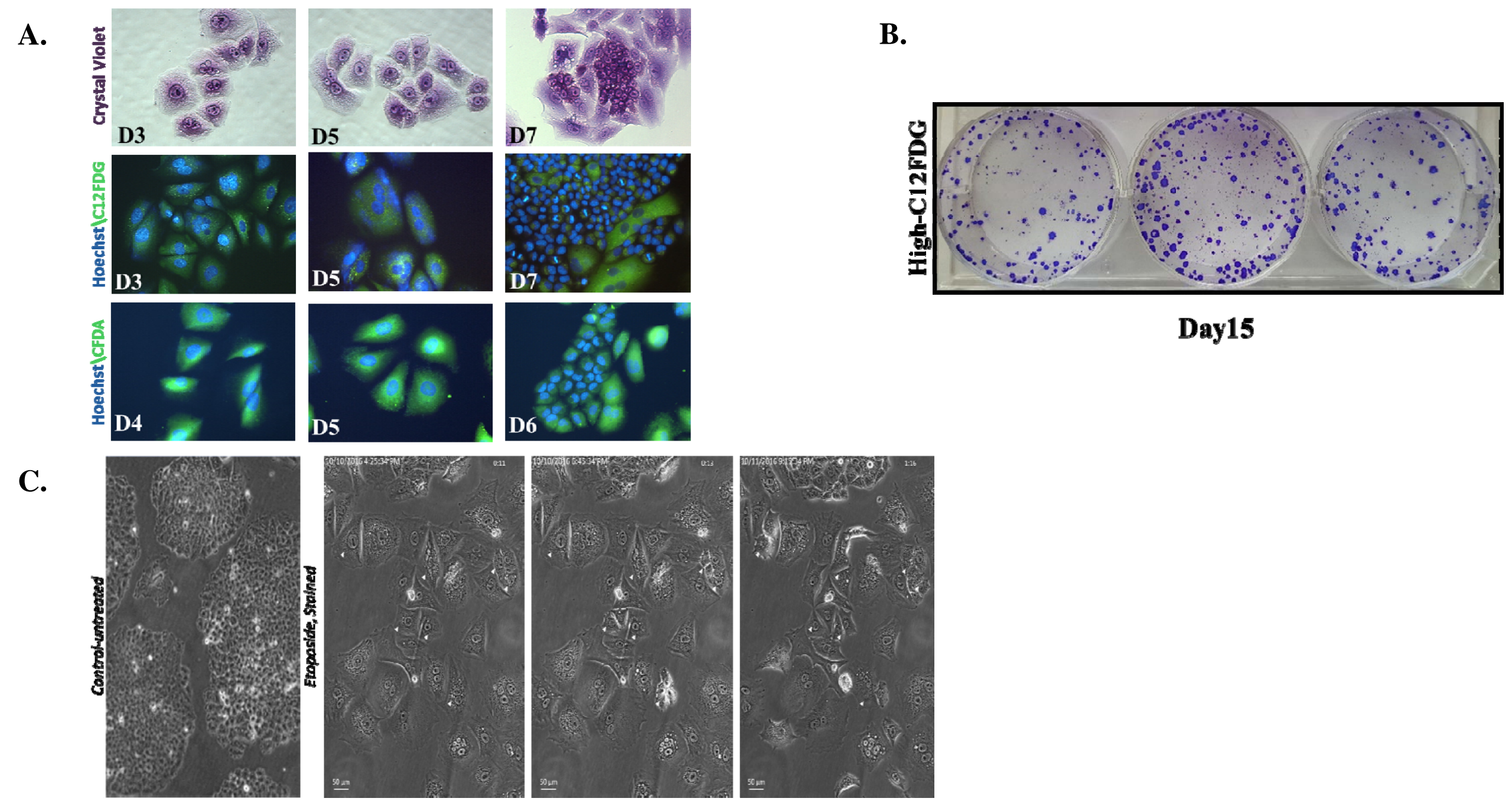

